\title{
Temporal dispersion in underwater laser communication links: closing the loop between model and experiment
}

\author{
Brandon Cochenour \\ Naval Air Warfare Center \\ Patuxent River MD 20670 \\ Email: brandon.cochenour@navy.mil
}

\author{
Alan Laux \\ Naval Air Warfare Center \\ Patuxent River MD 20670
}

\author{
Linda Mullen \\ Naval Air Warfare Center \\ Patuxent River MD 20670
}

\begin{abstract}
An elevated interest in underwater optical communications has resulted in the development of numerous theoretical models to predict both signal attenuation and bandwidth (pulse spreading) for underwater optical links. Few, if any, of these models have been experimentally validated, owing mostly to the difficulty in measuring temporal dispersion in these challenging environments. In this work, we begin to close the loop between theory and experiment, by validating a numerical Monte Carlo model against experimental data. Channel attenuation and frequency response is measured out to $1 \mathrm{GHz}$ over $\sim 20$ attenuation lengths in a line-of-sight configuration.
\end{abstract}

\section{INTRODUCTION}

The study of laser propagation underwater dates back to the 1970's [1], [2]. In the past 15 years, coincident booms in the fields of autonomous and remotely operated vehicles as well technological advances in laser source and receiver components have made underwater optical communications both attractive and possible.

As with any wireless communications link, it is desirable to have a theoretical understanding of the communications channel in order to predict and optimize performance. This is especially true undersea, where small changes in inherent optical properties (IOP's) may result in large changes in link performance. Specifically, the IOPs of scattering and absorption influence both spatial dispersion (beam spreading) as well as temporal dispersion (pulse spreading). Understanding the propagation characteristics of the channel are therefore necessary to optimize a link for a given range, bandwidth, pointing accuracy, and geometry (line-of-sight, non-line-ofsight, etc.).

Unfortunately, mathematically describing optical propagation in seawater is not a straightforward task. A complete mathematical description is given by the Radiative Transfer Equation (RTE), which is a complex integro-differential equation of several variables in space and time [3]. Solutions to the RTE provide a link designer with both the spatial and temporal properties of the channel required for predicting both the received signal level and the maximum supported bandwidth or data rate. Finding tractable solutions to the RTE is no

U.S. Government work not protected by U.S. copyright trivial task, and typically falls into two categories: analytical or numerical.

Analytical expressions are often desired for computational simplicity. However the RTE often does not provide manageable solutions unless certain approximations are made. This can take the form of assumptions in how light scatters [4][6] or in assuming a mathematical form of the final solution, which may not be valid in all conditions [7], [8]. For this reason numerical models using Monte Carlo methods, which rely on statistical distributions of the IOPs to ray-trace photons through a medium can be an attractive alternative. Numerical models however may require significant processing time, particularly in turbid environments, where a large number of photons may be need to be simulated in order to obtain statistical accuracy. Johnson et. al. [9] provides an excellent review of modeling approaches for further comparison and analysis.

While there has been an increase in the number of models reported in the literature, the vast majority of these models have limited, if any, experimental validation. Additionally, many use approximations for important IOPs that describe the angular distribution of scattering events, which may lead to performance prediction errors [10]. In this work, we aim to close the loop between experiment and theory though experimental validation of a Monte Carlo model. Specifically, we examine the channel attenuation and frequency response out to $1 \mathrm{GHz}$ over 20 attenuation lengths in a line of sight configuration. The scattering function is measured in-situ in order to increase accuracy and relevancy of the model results. The sensitivity of these link metrics to water optical properties and system variables are briefly examined.

\section{MODEL INPUTS AND DESCRIPTION}

The Monte Carlo (MC) technique assumes that the known probability of occurrence of each separate event in a sequence of events can be used to estimate the probability of the occurrence of the entire sequence. This is achieved by deriving the sample functions of each random variable in the process. In our specific case, the known probability of path lengths traveled between scattering events, and the angle of scattering 
after each event, affords the determination the spatial, angular, and temporal distribution of light at any point within the simulation volume. For additional background, Mobley [11] provides an introduction to Monte Carlo fundamentals and sampling function derivation in the context of underwater photon propagation. The codes in this study are based on that of Laux et. al. [12], and are segmented into two components. The first component employs said probability distributions to determine the light field at a given plane within the simulation volume, while the second component determines the spatial, angular, and temporal distributions at the given location given a particular receiver characteristic.

The propagation routine requires knowledge of the water channel IOPs, namely the absorption coefficient, $a\left(\mathrm{~m}^{-1}\right)$ the scattering coefficient, $b\left(\mathrm{~m}^{-1}\right)$, and the scattering phase function $p(\theta)$. The sum of absorption and scatteirng is the total attenuation coefficient, $c\left(\mathrm{~m}^{-1}\right)$. We will currently ignore the wavelength dependence of these coefficients, noting that in this work all values are given at $532 \mathrm{~nm}$. It is well known that non-scattered light attenuates with physical distance, $z$, according to Beer's Law, or $P(z)=P_{0} e^{-c z}$. The product $c z$ is referred to as the attenuation length. The scattering albedo is given by $\omega_{0}=b / c$. The scattering phase function, $p(\theta)$, can be interpreted as a probability distribution of scattering into a given solid angle $d \Omega$ in the $\theta$ direction (assuming symmetry in the $\phi$ direction). Obviously, the phase function is an important characteristic of the underwater optical channel, as it governs the directions of scattered photons which in turn influences both spatial and temporal dispersion of the communications signal.

Using the above optical properties, the propagation routine launches a photon from the source. A random path length is sampled from an exponential distribution (governed by the scattering coefficient), and the photon is propagated that distance along it's current direction. If the photon is still within the scattering volume, the next scattering direction is determined by randomly sampling the phase function (assuming azimuthal symmetry), and then transformed to the global coordinate system. Propagation continues until the photon exits the scattering volume. Those that reach the receiver collector are saved to a file along with details regarding their path length traveled, position on the collector, and angle of arrival. Non-scattered photons reaching the collector are also logged as well as the number of photons launched to ensure proper scaling of the received signal amplitude in the processing routine. This approach allows the user to investigate various receiver apertures, positions, and fields of view in post processing without the need to re-run the simulation. For each turbidity, the Monte Carlo procedure is generally run until $10^{6}$ scattered photons reach the collector to ensure sufficient statistics.

The processing routine implements a photodetector of userdefined aperture and field of view at a certain location on the collector plane. Photons that do not coincide with the detector aperture and location are ignored, and those within the detector are then weighted according to the receiver FOV.
A final weighting is added to account for absorption over the total path length traveled. The remaining non-zero weighted photons then represent those received by a photodetector of particular position, aperture, and FOV. Time-of-arrivals are then calculated for the received photons, and a histogram is formed to obtain the impulse response. An FFT of the impulse response gives the theoretical modulation frequency response of the channel.

\section{EXPERIMENT}

In this work, we use the measurement technique described by Mullen and Cochenour [13]-[16] that provides increased sensitivity, dynamic range, and signal-to-noise over a wide band of frequencies (DC - $1 \mathrm{GHz}$ ) compared to the inherently wideband time domain approach which aims to measure the spreading of a very short pulse.

We use a custom continuous wave $(\mathrm{CW})$ fiber laser source from AdvR (Bozeman, MT) to provide the necessary channel input. The laser is seeded with a $1064 \mathrm{~nm}$ laser diode, which is intensity modulated by a fiber coupled Mach Zehnder modulator. The intensity modulated infrared light is then fiber amplified, and then frequency doubled to $532 \mathrm{~nm}$. The result is a high frequency (up to $1 \mathrm{GHz}$ ) $\mathrm{CW}$ intensity modulated green beam with $500 \mathrm{~mW}$ of average power. The beam aperture is $0.7 \mathrm{~mm}$ with a $0.01^{\circ}$ divergence. A photomultiplier tube (PMT) from Photonis (Sturbridge, MA) designed especially for high frequency operation is used to recover the modulated optical signal, and is placed at the opposite end of a $1 \mathrm{~m} \mathrm{x} 1 \mathrm{~m}$ x 3.66m water tank. Receive optics include a 2-inch f/2 lens and variable aperture, resulting in a 9 degree (full angle) field of view (FOV). The AC and DC components are measured simultaneously with a spectrum analyzer and multimeter, and the normalized channel response is given by the Modulation Depth, defined here as $M D(f)=10 \log \left[V(f)_{A C} / V_{D C}\right]$. The system response is measured in clear water as a function of frequency, and used to calibrate the subsequent data taken at increasing turbidities.

Water turbidity is controlled via the addition of Equate, a commercial antacid. The absorption and total attenuation coefficients are measured in-situ with an AC-9 transmissometer (WET Labs, Philomath, OR). In-situ data from the experiment shows that the scattering albedo of Equate is $\omega_{0}=b / c=0.89$. The scattering phase function was measured in-situ with the LISST-VSF instrument (Sequoia Scientific, Bellevue, WA) and is shown in Figure 1. The measured IOP's are then used as inputs to the model.

\section{RESUlTS}

The Monte Carlo output is compared to experiment in Figure 2. Here, we plot the spatial and temporal dispersion by viewing the amplitudes of both the $\mathrm{AC}$ and $\mathrm{DC}$ components, as well as the normalized channel response (i.e. - Modulation Depth), as a function of attenuation length for $100 \mathrm{MHz}, 500$ $\mathrm{MHz}$, and $1 \mathrm{GHz}$. Note that there are two distinct features in the results. At low attenuation lengths, both the DC and AC amplitudes attenuate at a rate similar to Beer's Law, and the 


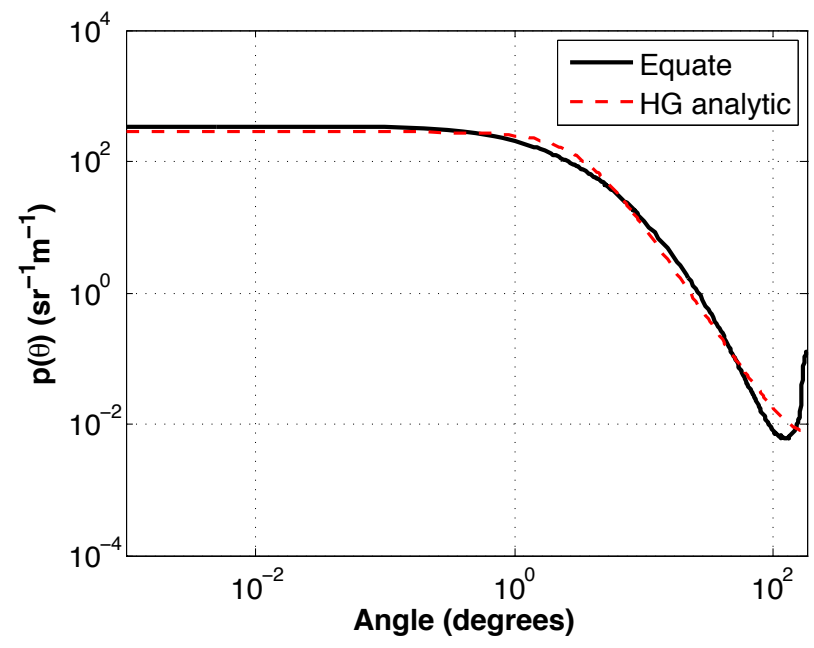

Fig. 1. Phase function of Equate antacid as measured by the LISST-VSF (black) and the analytic Henyey-Greenstein phase function (red).

Modulation Depth remains near unity. This suggests that nonscattered or minimally scattered light dominates at the receiver. At higher attenuation lengths the rate of attenuation slows, as multiply scattered light scatters back into the receiver aperture. The extra loss observed in the AC components are due to destructive interference arriving from path length differences between multiply scattered photons. This interference results in a reduction of channel bandwidth, and is analogous to intersymbol interference.

Discrepancies between model and experiment are pronounced in the multiple scattering region for both spatial and temporal dispersion. This suggests either errors within the Monte Carlo codes or inaccuracies in the inputs to the model (e.g. - the water optical properties or system parameters). To investigate the sensitivity to water optical properties, we draw on conclusions from previous experimental investigations. For example in [14], [17], it was shown that the scattering albedo influences the amount of scattered light collected in the multiple scattering regime, and hence the magnitude of temporal dispersion. In [14], [15], it was shown that changes in scattering phase function can result in a change in the rate of modulation loss as a function of attenuation length. Receiver FOV has influence on both spatial and temporal as well [15]. Cox and Muth performed a trade-off analysis of several system and environmental variables via Monte Carlo modeling as well, though the model was never validated [18].

To briefly illustrate the potential influence of these parameters on the results, we use the model to consider the following alternative cases:

Case 1: Presumed experimental baseline $\left(\omega_{0}=0.89, \mathrm{FOV}\right.$ $=9^{\circ}$, Equate phase function)

Case 2: No absorption $\left(\omega_{0}=1, \mathrm{FOV}=9^{\circ}\right.$, Equate phase function)

Case 3: $2 x$ FOV $\left(\omega_{0}=0.89, \mathrm{FOV}=18^{\circ}\right.$, Equate phase function)

Case 4: Alternative phase function $\left(\omega_{0}=0.89, \mathrm{FOV}=9^{\circ}\right.$,
Henyey-Greenstein phase function)

In Case 4, we use the Henyey-Greenstein approximation as the alternative phase function and set the average cosine parameter, $g$, to be the same as measured from Equate $(g=0.94)$. The phase function is plotted along with Equate in Figure 1.

The results of all 4 cases, along with the experimental data, are shown for $1 \mathrm{GHz}$ in Figure 3. Changing the amount of absorption appears to have the most significant effect on both spatial and temporal dispersion. The result is consistent with previous experimental observations mentioned above, as multiply scattered light now contributes more to the overall received signal rather than being attenuated by absorption events. The result is a higher amplitude and more temporal dispersion.

Including absorption losses but increasing the FOV by a factor of 2 results in the collection of more scattered light, but there is virtually no appreciable change in temporal dispersion. This suggests that for the given geometry, while there may be more scattered light collected at larger incident angles, they have not accumulated any appreciable path length differences to cause additional loss of modulation depth.

Finally, Figure 3 also shows that a change in scattering function results in the collection of slightly more scattered light at high turbidities. However in this case, there is less temporal dispersion. This suggests that even with the same average cosine as Equate, the subtleties in phase function shape are still important. We posit that there is a slightly higher probability of scattering at smaller forward angles overall in the HG phase function, which keeps scattered light closer to the beam axis. This results in the increase of light collected, but a decrease in path length differences (resulting in better modulation depth). The results are in qualitative agreement with previous experimental studies, however quantifying the exact effect of phase function on temporal dispersion is a subject of future study

Finally it should be mentioned that link range and aperture were not considered in this analysis, and are likely to have a significant impact on the above trends as well.

\section{SUMMARY}

We have presented an experimental validation of a numerical Monte Carlo model for predicting spatial and temporal dispersion in underwater optical communication links. Results show the sensitivity of model predictions to variations in certain channel and system parameters. It is likely that a combination of these variables are responsible for differences between model and experiment. Future efforts will focus on how to best characterize the experimental environment to provide the most accurate inputs to the model. 


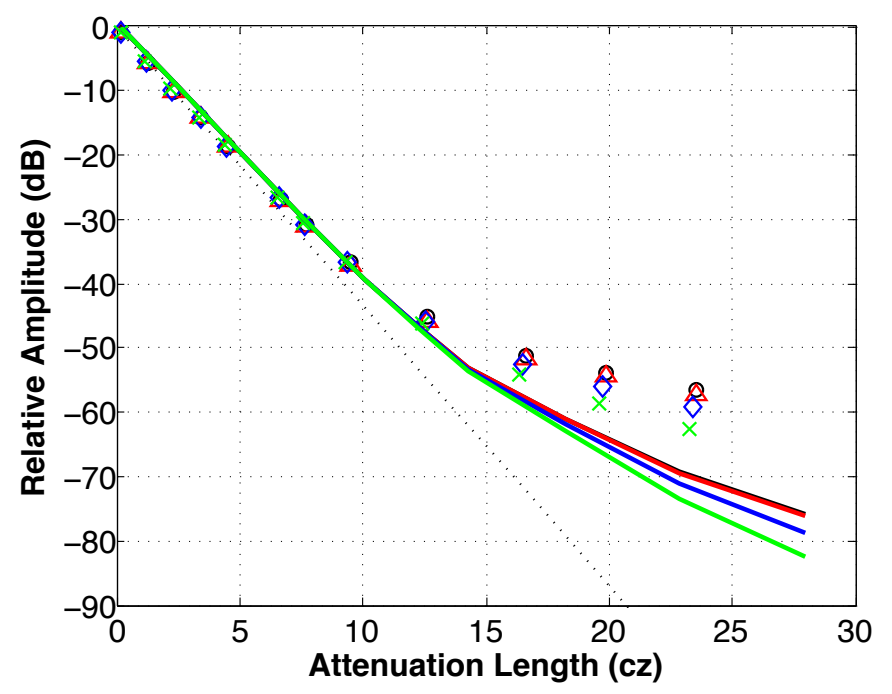

(a)

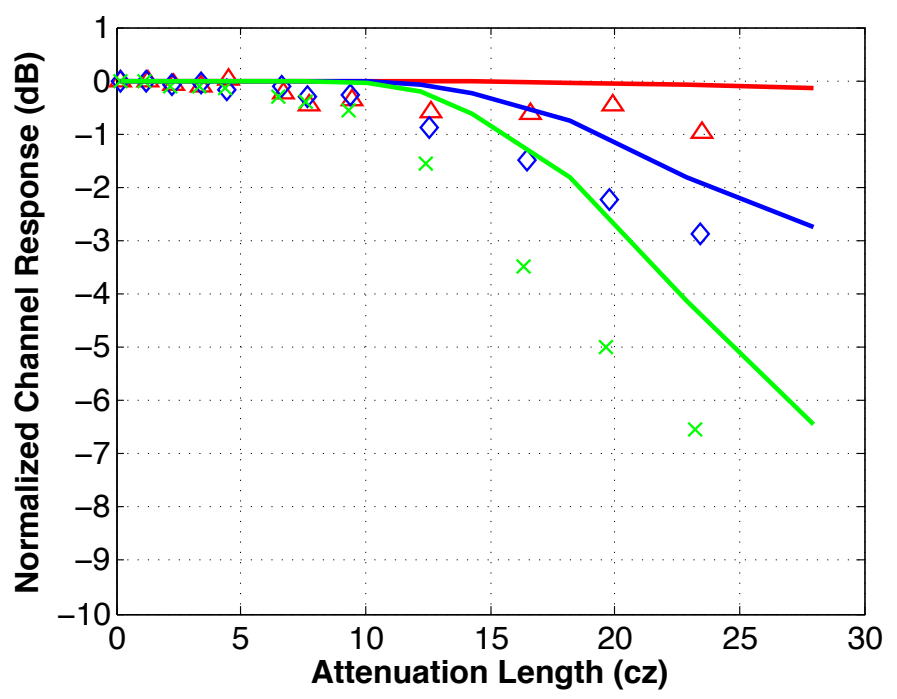

(b)

Fig. 2. Comparison of the (a) attenuation and (b) Modulation Depth between experiment (points) and numerical Monte Carlo model (solid line). Results are shown at DC (black), $100 \mathrm{MHz}$ (red), $500 \mathrm{MHz}$ (blue) and $1 \mathrm{GHz}$ (green).

\section{REFERENCES}

[1] S. Duntley, "Underwater Lighting by Submerged Lasers and Incandescent Sources," San Diego, CA, Tech. Rep., 1971.

[2] T. Petzold, "Volume Scattering Functions for Seleted Ocean Waters," San Diego, CA, Tech. Rep., 1972.

[3] S. Chandrasekhar, Radiative Transfer. New York: Dover Publications Inc, 1960.

[4] W. Wells, "Loss of resolution in water as a result of multiple small-angle scattering," Journal of the Optical Society of America, vol. 59, no. 6, pp. 686-691, 1969.

[5] — , "Theory of small angle scattering," Brussels, Belgium, Tech. Rep. Optics of the Sea, 1973.

[6] B. Cochenour, L. Mullen, and A. Laux, "Characterization of the BeamSpread Function for Underwater Wireless Optical Communications Links," Oceanic Engineering, IEEE Journal of, vol. 33, no. 4, pp. 513521, 2008.

[7] I. Katsev, E. Zege, A. Prikhach, B. Cochenour, and L. Mullen, "Propagation of the amplitude-modulated beam though seawater: comparison of

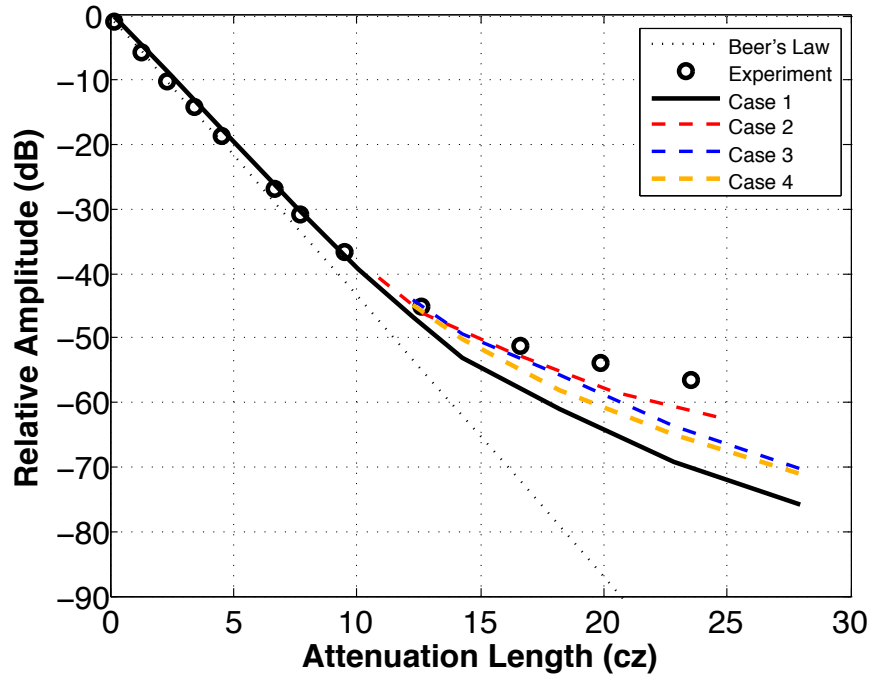

(a)

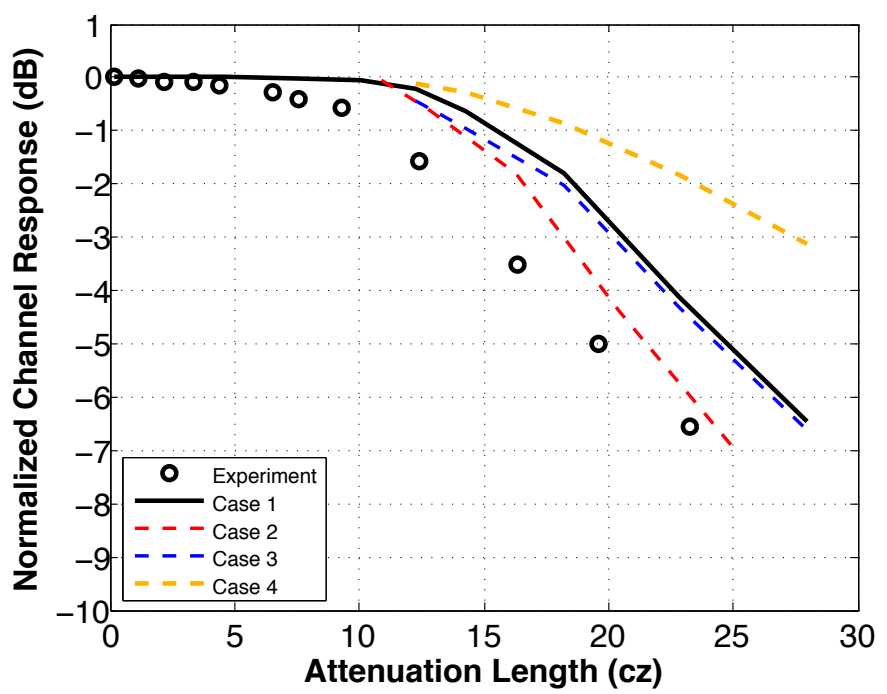

(b)

Fig. 3. The influence of water optical properties and system variables on the (a) amplitude, and (b) modulation depth at $1 \mathrm{GHz}$.

theory to experiment," in Current Problems in Optics of Natural Waters, St. Petersburg, 2013, pp. 128-132.

[8] — , "Demodulation of amplitude-modulated beams propagaing through seawaters," Fundamental and Applied Hydrophysics, vol. 8, no. $1,2015$.

[9] L. Johnson, R. Green, and M. Leeson, "A survey of channel models for underwater optical wireless communication," in Optical Wireless Communications (IWOW), 2013 2nd International Workshop on. IEEE, 2013, pp. 1-5.

[10] B. M. Cochenour and A. E. Laux, "Experimental validation of a Monte Carlo model for determining the temporal response of the underwater optical communications channel," SPIE Defense + Security, vol. 9459, pp. 94 590A-94 590A-9, May 2015.

[11] C. Mobley, Light and Water. San Deigo, CA: Academic Press/Elsevier Science, 1994.

[12] A. Laux, R. Billmers, L. Mullen, B. Concannon, J. Davis, J. Prentice, and V. Contarino, "The abc's of oceanographic lidar predictions: a significant step toward closing the loop between theory and experiment ,"Journal of Modern Optics, vol. 49, pp. 439-451, 2002. 
[13] L. Mullen, A. Laux, and B. Cochenour, "Propagation of modulated light in water: implications for imaging and communications systems," Applied Optics, vol. 48, no. 14, pp. 2607-2612, 2009.

[14] L. Mullen, D. Alley, and B. Cochenour, "Investigation of the effect of scattering agent and scattering albedo on modulated light propagation in water," Applied Optics, vol. 50, p. 1396, Apr. 2011.

[15] B. Cochenour, L. Mullen, and J. Muth, "Temporal Response of the Underwater Optical Channel for High-Bandwidth Wireless Laser Communications," IEEE Journal of Oceanic Engineering, pp. 1-26, 2013.

[16] B. Cochenour, "Experimental Measurements of Temporal Dispersion for Underwater Laser Communications and Imaging," Ph.D. dissertation, North Carolina State University, Raleigh, NC, Dec. 2012.
[17] B. Cochenour, L. Mullen, and J. Muth, "Effect of scattering albedo on attenuation and polarization of light underwater," Optics Letters, vol. 35 , no. 12 , pp. 2088-2090, 2010.

[18] W. Cox and J. Muth, "Simulating channel losses in an underwater optical communication system," JOSA A, vol. 31, 2014. 